\title{
Scimitar Syndrome: Infra-Diaphragmatic Form a Novel Surgical Approach
}

\author{
Carlos Riera-Kinkel ${ }^{1}$, Sesbania Bocanegra-Flores ${ }^{1}$, Martin Rosas-Peralta² ${ }^{2}$, Gabriela Borrayo-Sánchez ${ }^{2}$ \\ David Roldan-Morales ${ }^{1}$, Jaime Salgado-Vázquez ${ }^{1}$, Alberto Ramirez-Castañeda ${ }^{1}$, Sebastián Izunza-Saldaña ${ }^{3}$, \\ Diana López-Gallegos ${ }^{4}$, Horacio Márquez-González ${ }^{4}$, Moisés Jiménez-Santos ${ }^{5}$, Lucelli Yáñez-Gutiérrez ${ }^{4}$ \\ ${ }^{1}$ Cardiothoracic Surgery Division, Cardiology Hospital, National Medical Center SXXI-IMSS, México \\ ${ }^{2}$ Programa "A Todo-Corazón", National Medical Center, XXI century IMSS, México City, Mexico \\ ${ }^{3}$ Anesthesia Department, Cardiology Hospital, National Medical Center SXXI-IMSS, México \\ ${ }^{4}$ Congenital Heart Disease Department, Cardiology Hospital, National Medical Center SXXI-IMSS, México \\ ${ }^{5}$ Radiology Department
}

Received: February 26, 2019; Accepted: May 24, 2019; Published: June 20, 2019

*Corresponding author: Hospital de Cardiología, National Medical Center Siglo XXI, Cuauhtémoc 330, Colonia Doctores, CP 03020 , Tel: 5255 56276927, E-mail: rierac7@gmail.com

\section{Introduction}

Scimitar syndrome is a complex malformation in which the main feature is a partial anomalous venous drainage of the right side toward the inferior vena cava or the portal system. There are anomalies associated in bronchopulmonary segmentation and vascular lung connections [1]. In 1836 in specimens of autopsy Cooper in London published the first description and later Chassinat in París [2, 3]. In 1949 Dotter et, al through angiocardiography shown the first clinical diagnosis where noted the particular appearance of the anomalous vein on x-ray of thorax [4]. Later Neill et, al in 1960 called the scimitar sign for describing this anomaly [5]. Drake and Lynch in 1950 performed the first surgical management of this disease [6]. Later the first physiological correction of anomalous venous drainage was published in 1956 by Kirklin et, al [7]. Finally Neill CA, et al. published the familial occurrence of Scimitar syndrome.

The objective of this report is to inform of a case successfully repaired using an exterior tube. We analyzed the current guidelines for diagnosis and management, particularly in the adult patient.

\section{Clinical Case}

Female 38 years of age, who presented a pulmonary murmur, it was detected at 13 years of age, without follow-up; in 2002 began to be studied by suspicion of pulmonary hypertension, her principal symptom was dyspnea with Class II of NYHA. In 2015 reset study protocol for congenital heart disease and moderate pulmonary arterial hypertension. Laboratory: $\mathrm{Hb} 13 \mathrm{~g} / \mathrm{dL}$, Hto $32 \%$, Leucos 6,000 mm3, platelets 203,000 mm3, Creatinine $0.59 \mathrm{mg} / \mathrm{dL}$ | BUN $12 \mu \mathrm{g} / \mathrm{dL}$ | Glucemia $88 \mathrm{mg} / \mathrm{dL}$ | INR 1.1, NT - PRO BNP: 235. Rx-Thorax and EKG are shown in Figure 1. The echocardiography report shown an anomalous pulmonary venous drainage and suggested a Scimitar syndrome Table 1. Surgical
Findings: Cardiomegaly grade II at the expense of right cavities, aorto-pulmonary ratio $1: 3$, extra cardiac anomalous venous drainage with venous duct in " $\mathrm{H}$ " which empties into the inferior vena cava, correlation with scimitar syndrome, right atrium dilated without atrial septal defect, without presence of any pulmonary drainage Figure 2, 3. A Dacron tube was used as extra cardiac conduit to make surgical repair Figure 4. After unclamping patient presented ventricular fibrillation which required three defibrillation shock of 10, 20 and 20 Joules reaching a normal rhythm; Cardiopulmonary Bypass time: 181 minutes; bleeding: $320 \mathrm{ml}$; recovered: $259 \mathrm{ml}$; blood products: one globular package and two fresh frozen plasma. After a week she was discharged without any complication. An angio-tomography performed later was able to shown the final result Figure 5.

\begin{tabular}{|c|c|}
\hline \multicolumn{2}{|c|}{ Table 1: Echocardiogram parameters } \\
\hline LA & $33 \times 31 \times 44 \mathrm{~mm}$ \\
\hline RA & $41 \times 48 \times 46 \mathrm{~mm}$ \\
\hline LVEF & $64 \%$ \\
\hline Septum & $10 \mathrm{~mm}$ \\
\hline Posterior Wall & $10 \mathrm{~mm}$ \\
\hline TAPSE & 18 \\
\hline Systolic Pulmonary Pressure & $75 \mathrm{mmHg}$ \\
\hline
\end{tabular}

\section{Discussion}

Developing early pulmonary venous sinus communicates through small channels with systemic veins of the embryo. This primitive drainage disappears when the common pulmonary vein of the left atrium is connected with such senous of this vein Agenesis determines the persistence of the first channels which causes different types of anomalous connection $[8,9]$. 


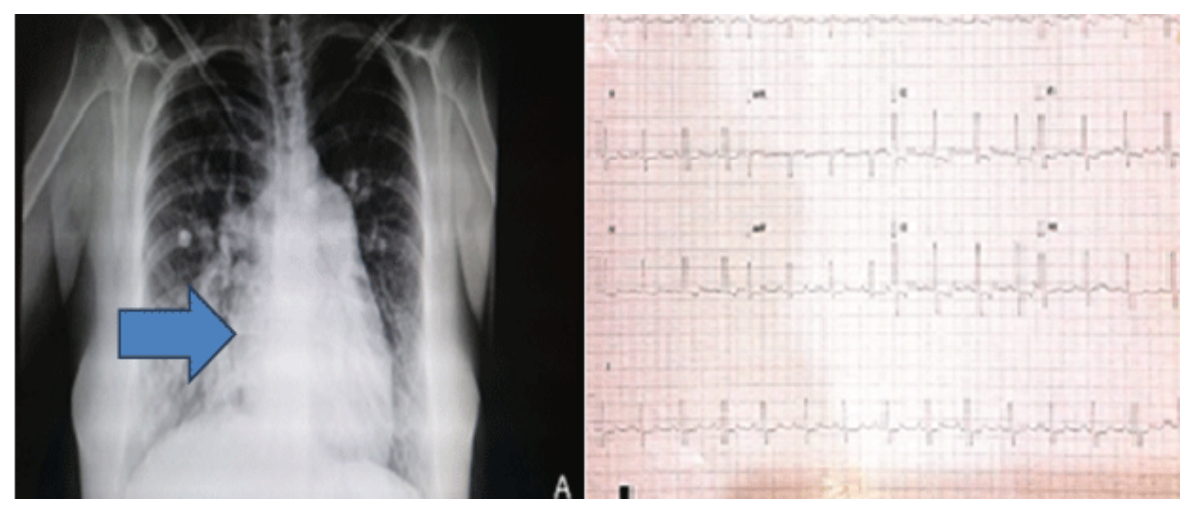

Figure 1: A: RX Chest : Describes the typical image of a scimitar in the right side margin B: EKG Sinus rhythm, $\mathrm{AQRS}+60^{\circ}$, Growth of right cavities and systolic overloading, growth of right atrium

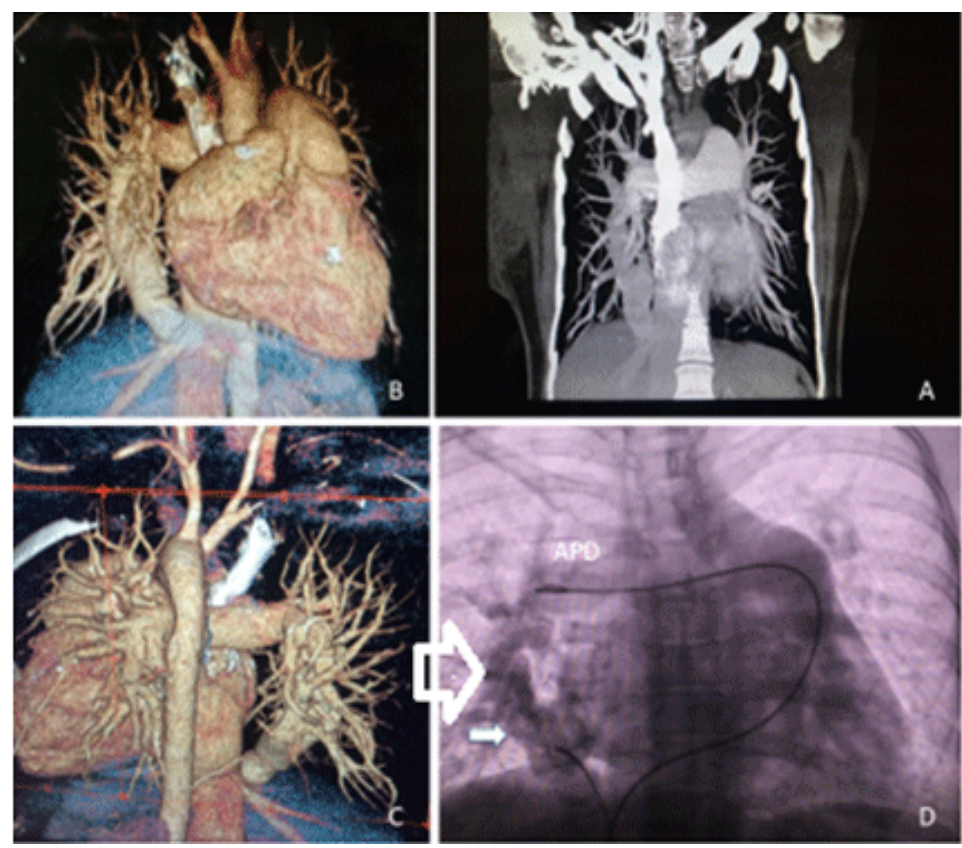

Figure 2: A: Angiotomography; B \& C: Third dimention Angiotomography, where the passage of the drainage through the diaphragm is shown, arriving at the suprahepatic vein, which is an event that takes place in very rare form; D: Cardiac catheterization. No cyanogen Congenital Heart Disease with anomalous venous drainage of the Pulmonary veins; Right upper and lower Pulmonary veins draining toward a collector (rear, right), which drains into the union of the inferior cava vein with right atrium; Left Pulmonary veins well-connected; LV normal with LVEF $60 \%$, LVDEP 10 mmHg; Mitral valve; Aortic components | Left Aortic Arch | Origin and normal distribution of coronary arteries; Dilated Pulmonary arch, Severe pulmonary hypertension $(90 \mathrm{~mm} \mathrm{Hg}$ ) which descending to $70 \mathrm{mmHg}$ after oxygen supplement. Arrow : right pulmonary branch (scimitar) 


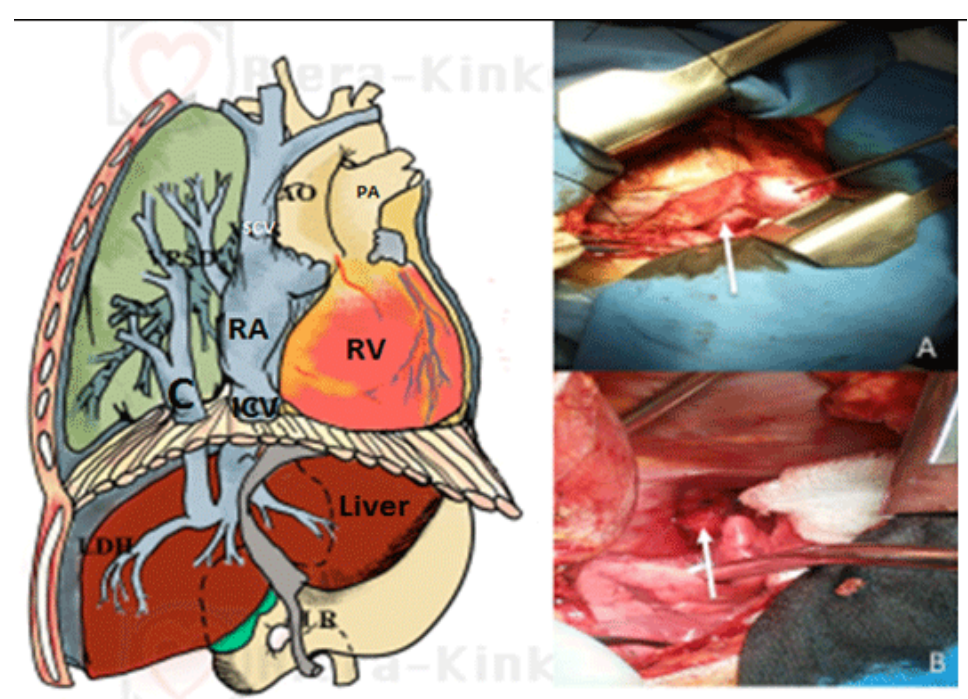

Figure 3: PA: Pulmonary Artery; AO: Aorta, SCV: Superior cava vein; RA: right atrium; ICV: Inferior Cava Vein; C: Scimitar, RV: Right Ventricle, LR: Round Ligament.

A \& B : Anomalous vein throwing diaphragm ( arrow Scimitar)
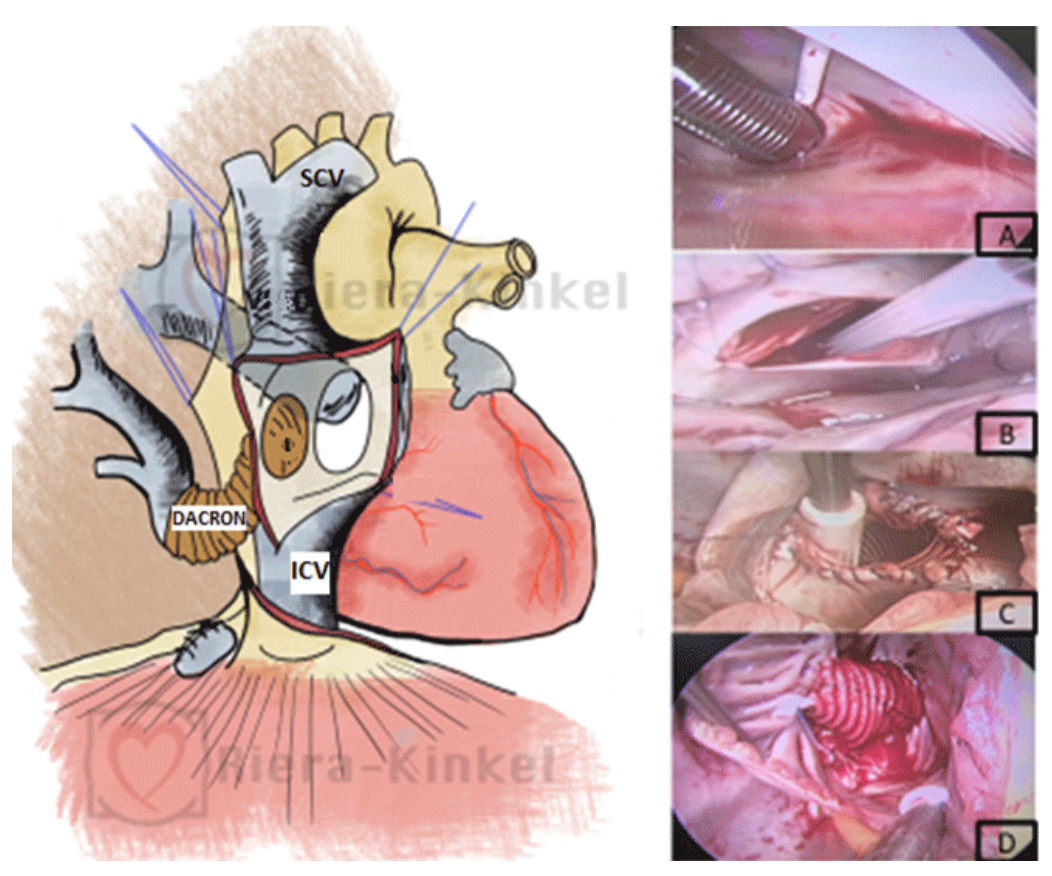

Figure 4: SCV: superior cava vein, ICV: Inferior cava vein. IAS: inter-atrial Septum A: Normal interatrial septum.

B \& C: right atrium-Dacron graft anastomosis

D: Formation of Dacron hemi tube ceiling 


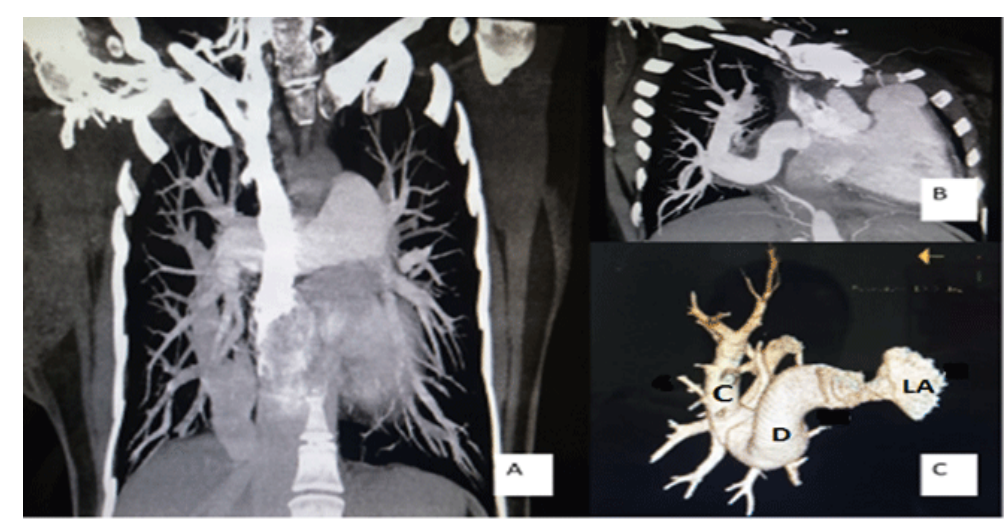

Figure 5: Post-surgery Angiotomography; A: Pre-surgery tomography; B: Post-surgery; C: 3D reconstruction. LA: left atrium, D: dacron graft , C: Scimitar

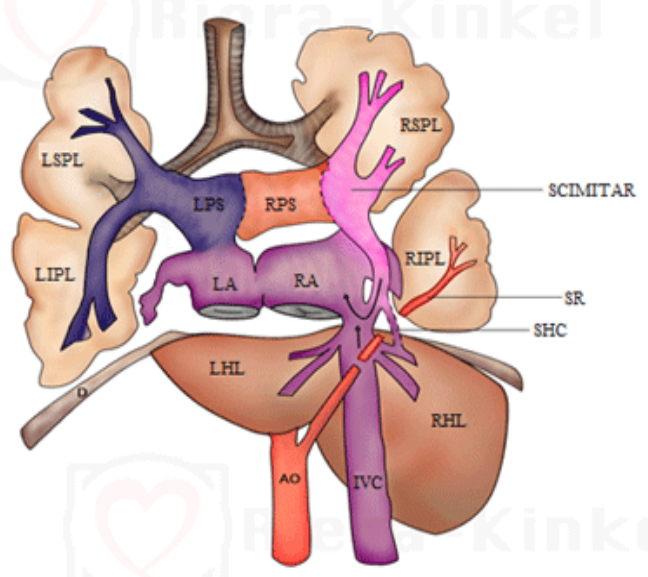

Figure 6: Posterior view of venous connection: LSPL, Left Superior Pulmonary Lobule; RSPL, Right Superior Pulmonary Lobule; LPS, Left Pulmonary Sinus; RPS, Right Pulmonary Sinus; LIPL, Left Inferior Pulmonary Lobule; LA, Left Atrium; RA, Right Atrium; SR, segment ramus; (pulmonary sequestration); SHC, Suprahepatic Communication; LHL, Left Hepatic Lobule; RHL, Right Hepatic Lobule; AO, Aorta; IVC, Inferior vena cava

Scimitar syndrome pulmonary venous sinus presents double connection, on the left with the left atrium and the right side with the supra-hepatic segment of the inferior vena cava, derived from the right vitelline vein of the embryo; This favors the separation of pulmonary venous sinus in two portions, left joins to the left atrium and right is continued with the curved manifold Figure 6.

The connection of the right pulmonary veins to level infradiaphragmatic either to the vena porta or inferior vena cava types that deviate from the scimitar syndrome. Partial anomalous pulmonary venous connection to the right atrium without curved manifold and right pulmonary hypoplasia should be excluded from the scimitar syndrome.

Scimitar syndrome is classified in three groups according to the age of the patients as it was suggested in a multicenter study of 122 patients with ages between 1 and 58 years old. The Group I correspond to adult patients without pulmonary hypertension $(\mathrm{PH})$ and with interatrial septal communication; group II, It has complex congenital abnormalities affecting the symptomatology and the natural history of this syndrome; Group III the child is characterized by severe and poor PH prognosis [10-13]. The most common symptoms present in all patients were breathing and progressive dyspnea $[11,12,14]$. In some cases the radiographic sign of the scimitar is not observed as a consequence of the cardiac dextrorotation or because of the venous collector is not curved or wide rather than straight, slim or multiple. This sign is presented by $70 \%$ of patients. It is due to hypoplasia of the right lung and it is absent in adults $[10,15]$.

The echocardiographic diagnosis had a good correlation with cardiac catheterization. Transthoracic echocardiography has more value in children than adults with this syndrome; and, in adults the diagnosis must be complemented with Transesophageal Echocardiogram to assess structures as the atrial septum position, the size, number, morphology and localization of the defects and the connection of the Pulmonary veins that cannot 
be well valued by Trans thoracic technique. Trans esophageal echocardiography is also useful for detecting obstructions in tubes placed for the replantation as happened in one of the cases studied was corroborated by resonance magnetica $[10,13,16]$.

It is very important to determine the levels of the supra connection for a better surgical management. A surgical alternative could be the re-implantation of the manifold to the rear wall of the left atrium without extracorporeal circulation, as Brown and collaborators carried out it [12, 14, 17-20].

\section{Conclusions}

On the basis of our case-study we concluded that scimitar syndrome is rare, whose diagnosis is performed in the majority of cases by echocardiography. With the development of techniques not only in some cases invasive catheterization diagnosis is complementary $[18,19]$. A correlation anatomoechocardiographic is useful because it shows the correspondence between the anatomical feature and its image diagnostic which confers a degree of precision to the diagnostic echocardiography, which is essential for the surgical decision, however computed, with three-dimensional reconstruction tomography, images, allowing perfectly provides the surgery planning.

Surgical treatment of scimitar syndrome during the pediatric age, allows direct anastomosis of the right inferior pulmonary vein to the left atrium, referring cases without cardiopulmonary bypass [20]. During the adult stage the flexibility of the tissue makes it more difficult this situation it is necessary to interpose a Dacron graft as it was met in this case.

Another important particularity of this case was the small left atrium, so it was necessary to perform the anastomosis of the dacron tube into the right atrium, and subsequently open the atrial septum, and place a Dacron tube in dome-shaped, allowing re-direct the flow to the left atrium.

\section{References}

1. Farnsworth AE, Ankeney JL. The spectrum of scimitar syndrome. J Thorac Cardiovasc Surg. 1974;68(1):37-42.

2. Cooper G. A case of malformation of the thoracic viscera consisting of imperfect development of the right lung and transposition of the heart. Med Gaz (London). 1836;18:600-604.

3. Chassinat R. Observation d' anomalies anatomiques remarquables de l'appareil circulatoire avec hepatocele congenitale, n'ayant donne lieu pendant la vie a aucun symptome particulier. Arch Gen Med. 1836;11:80-85.

4. Dotter CT, Hardisty NM, Steinberg I. Anomalous right pulmonary vein entering the inferior vena cava. Two cases diagnosed in life by angiocardiography and cardiac catetherization. Am J Med Sci. 1949;218(1):31-36.

5. Neill CA, Ferenca C, Sabiston DC, Shelton JC. The familial ocurrence of hypoplastic right lung with systemic arterial supply and venous drainage:"Scimitar syndrome". Bull Johns Hopkins Hosp. 1960;107:121.
6. Drake EH, Lynch JP. Bronchiectasis associated with anomaly of the right pulmonary vein and right diaphragm. J Thorac Surg. 1950; 19(3):433-437.

7. Kirklin JW, Ellis H, Wood EH. Tratment of anomalous pulmonary venous connection in association with interatrial communications. Surgery. 1956;39(3):389-398.

8. Auer J. Development of the pulmonary vein and its mayor variations. Anat Rec. 1948;101:851-895.

9. Van Praagh R, Corsini I. Cor Triatriatum: Pathologic anatomy and a consideration of morphogenesis based on 13 post mortem cases and a study of normal development of the pulmonary vein and atrial septum in 83 human embryos. Am Heart J. 1969;78(3):397-405.

10. Geggel RL. Scimitar Syndrome associated with partial anomalous pulmonary venous connection at the supracardiac, cardiac and infracardiac levels. Pediatr Cardiol. 1993;14(4):234-237.

11. Dupuis C, Charaf L, Breviere GM, Abou P, Remy-Jardin M, Helmius G. The "Adult" form of the Scimitar Syndrome. Am J Cardiol. 1992; 70(4):502-507.

12. Salazar J. Scimitar Syndrome: Five cases examined with TwoDimensional and Doppler Echocardiography. Pediatr Cardiol. 1995;6(6):283-286

13. Depuis C, Charaf L, Breviere GM, Abou P. "Infantile" form of the scimitar syndrome with pulmonary hypertension. Am J Cardiol. 1993;71(15):1326-1330.

14. Cobanoglu A, Hanlon JT, Combs DT, Starr A. Combined intracardiac and extracardiac repair of scimitar syndrome with anomalous pulmonary veins to both cavae. J Thorac Cardiovasc Surg. 1984;88(1):141-146.

15.Idris MT. Diagnostic Aid of Transesophageal Echocardiography in an Adult Case of Scimitar Syndrome: Confirmation of the Findings at Surgery and Review of the literature. J Am Soc Echocardiogr. 1998;11(4):387-392.

16. Shibuya K, Smallhorn JE, McCrindle BW. Echocardiographic clues and accuracy in the diagnosis of Scimitar Syndrome. J Am Soc Echocardiogr. 1996;9(2):174-181.

17. Brown JW, Ruzmetov M, Minnich D, Vijay P, Edwards CA, Uhlig PN, et al. Surgical management of scimitar syndrome: An alternative approach. J Thorac Cardiovasc Surg. 2003;125(2):238-245.

18. Inoue T, Ichihara M, Uchida T, Sakai Y. Three-Dimensional computed tomography showing partial pulmonary venous connection complicated by the Scimitar Syndrome. Circulation. 2002;105(5):663.

19. Rose C, Vosshenrich R. Incomplete Scimitar Syndrome. Cardiol Young. 2002:12(4);389-390.

20. Gil-Jaurena JM, Castillo Rafael, Sarria Esteban, Gonzalez Mayte. Asian Cardiovascular and Thoracic Annals. 2014;22(3):353-355. 